\title{
Fabrication and preliminary testing of hydroxylammonium nitrate (HAN)-based ceramic microthruster for potential application of nanosatellites in constellation formation flying
}

\author{
Kalaimani Markandan ${ }^{1} \cdot$ Zhe Zhang $^{2} \cdot$ Jitkai Chin $^{3}$ (D) Kean How Cheah ${ }^{4} \cdot$ Hai-Bin Tang $^{5}$
}

Received: 19 December 2018/Accepted: 12 May 2019/Published online: 23 May 2019

(C) The Author(s) 2019

\begin{abstract}
Constellation formation flying of nanosatellites $(<10 \mathrm{~kg})$ is becoming a trend for future space exploration missions. Liquid chemical micropropulsion that can provide high thrusting force for high $\Delta V$ orbital transfer is a crucial hardware component for successful constellation re-positioning. However, liquid chemical micropropulsion system dedicated for these nanosatellites still not currently available in the market. In this study, a microthruster was fabricated using alumina toughened zirconia (ATZ) as structural material and yttria-stabilised zirconia-graphene (YSZ-Gr) as electrode material. The device has a dimension of $1.7 \mathrm{~cm}$ (length) $\times 1.3 \mathrm{~cm}$ (width) $\times 0.5 \mathrm{~cm}$ (thickness) and weighing only 6.18 grams, comprising of five major components such as propellant reservoir, injector, electrodes, micronozzle and reaction chamber. Material characterization shows that both materials have negligible weight loss and small reduction in material hardness and fracture toughness after 20 thermal-vacuum cycles. Moreover, results from dynamic mechanical analysis (DMA) ascertain that addition of graphene significantly enhanced the damping behaviour of the zirconia composite. Electrolytic decomposition of hydroxylammonium nitrate (HAN) solution in the reaction chamber has been demonstrated with maximum thrust of $180.53 \mathrm{mN}$ at a propellant flow rate of $60 \mu \mathrm{l} / \mathrm{s}$. The preliminary results suggest that the HAN-based ceramic microthruster could potentially be used as primary propulsion for nanosatellites in constellation formation flying.
\end{abstract}

\section{Introduction}

In recent years, formation flying of nanosatellites $(<10 \mathrm{~kg})$ have attracted enormous attention from space community because of advantages such as short development time, low life cycle cost, reduced risk of mission failure, as well as versatility to changing mission goals

Jitkai Chin

J.Chin@hud.ac.uk

1 Department of Chemical and Environmental Engineering, University of Nottingham Malaysia Campus, Semenyih, Malaysia

2 School of Instrument and Optoelectronic Engineering, Beihang University, Beijing 100191, China

3 Department of Applied Sciences, University of Huddersfield, Huddersfield, West Yorkshire, UK

4 School of Engineering and Physical Sciences, Heriot-Watt University Malaysia, Putrajaya 62200, Malaysia

5 School of Space and Environment, Beihang University, Beijing 100191, China
(Kristiansen and Nicklasson 2009; Hu et al. 2015; Sun et al. 2017). In depth review on the formation control of nanosatellites, satellite formation flying, distributed satellite systems, and fractionated satellite formation have been reported previously (Liu and Zhang 2018; Chong et al. 2016).

Formation flying of satellites can be categorized into trailing, cluster and constellation. The fundamental success of satellite formation flying lies on the relative spatial and temporal positioning of each satellite in the formation, which is susceptible to unpredictable and dynamic space environment. For constellation formation flying, constant orbital correction and orbit re-phasing are required. For instance, orbit re-phasing is necessary if one of the satellites in the constellation fails, others may re-position themselves to ensure continuity of the mission. For an interplanetary mission, a swarm of nanosatellites could be designed to perform a series of risky manoeuvres, such as orbiting and landing on an asteroid while the 'mothercraft' remain on orbit to receive and transmitting data from landed space probe using high gain antenna. Requirements for these high $\Delta V$ scenarios are highly dependent on the 
mission while the choice of propulsion concept depends on the available time to conduct such manoeuvre (Mueller et al. 2008). High specific impulse electric micropropulsion is suitable for long duration mission. Cold gas microthruster or chemical microthruster with moderate specific impulse but high thrust-to-weight is ideal for rapid orbital transfer.

Cold gas thruster has been demonstrated as the feasible propulsion concept for the rapid $\Delta V$ applications. SNAP-1, a $6.5 \mathrm{~kg}$ nanosatellite, developed by University of Surrey is the first nanosatellite carrying cold gas thruster (Underwood et al. 2003) to perform inspection manoeuvres to another satellite in orbit. The separation between SNAP-1 and Tsinghua-1 (target microsatellite) has successfully been reduced from 15,000 to $2000 \mathrm{~km}$.

Nevertheless, the low specific impulse of cold gas thruster has limited total $\Delta V$. Chemical propellant microthruster offers higher specific impulse and thus higher total $\Delta V$. Hydrazine is a commonly used chemical propellant due to its heritage, such as GRAIL mission where two microsatellites used to measure moon gravity (EdwardsStewart 2013).

However, the high handling cost of the toxic hydrazine propellant is a drawback which does not favour nanosatellite applications. Electrolytic ignition of HAN solution is a promising approach for application in micropropulsion system. Previous study has demonstrated a complete combustion of HAN solution inside a $0.82 \mathrm{~mm}^{3}$ volume of combustion chamber produced $150 \mathrm{mN}$ of thrust with deposited electrical energy as low as $1.9 \mathrm{~J}(\mathrm{Wu}$ and Yetter 2009). A combination of cold gas and ADN green propellant thrusters have been demonstrated in the PRISMA rendezvous mission (Persson et al. 2010). Green ionic propellant, e.g. $\mathrm{ADN}$ and $\mathrm{HAN}$, is a promising replacement of hydrazine for chemical micropropulsion system.

Although extensive research work focusing on formation coordination (Ren and Sorensen 2008) and control methods (Cai et al. 2017; Zhang and Liu 2018) have been carried out, developing microthruster hardware specifically for nanosatellites in formation flying has only been reported recently, in which a low power micropropulsion system based on thermal decomposition of an inert chemical blowing agent (CBA) capable of delivering thrust of $1 \mu \mathrm{N}$ was reported (Romero-Diez et al. 2018).

Although specific requirement of green liquid propellant for nanosatellites in constellation formation flying have not been reported, several criteria for development of the microthrusters from literatures can be summarised as the following:

1. Provide mili-Newton level of thrust for rapid manoeuvre.
2. Environmentally friendly propellant-non-toxic, clean combustion by-products and easy to handle.

3. Compact system, i.e. small in size and light in weight for high thrust-to-weight ratio.

While green propellants are promising alternatives, their high combustion temperature poses stringent requirements on thermo-mechanical properties of the structural material. In recent years, advanced ceramic composites has been poised as the potential structural material for next generation liquid propellant or cold gas microthrusters (Lekholm et al. 2013; Cheah and Chin 2011, 2012, 2013). These ceramics are tolerant to high temperatures, lower heat conductivity, exceptional thermal shock resistance and chemically resistant (Schneider et al. 2008; Nevarez-Rascon et al. 2009; Bartolomé et al. 2016). Compared to stainless steel and siliconbased material, fabrication of microthruster using high temperature co-fired ceramic (HTCC) technique (Cheah and Low 2014) is relatively simple, and completed with less expensive equipment. However, HTCC are prone to crack after firing at high temperature (Khaji et al. 2016) and not tested in space simulated environment.

In this paper, a high $\Delta V$ HAN-based microthruster was fabricated using Alumina Toughened Zirconia (ATZ) ceramic and Yytria Stabilised Zirconia-Graphene ceramic composite as the structural and electrode materials, respectively. The microthruster is a scale-up based on Hydrazine mili-Newton Thrust (HmNT) developed by NASA Jet Propulsion Laboratory (Mueller et al. 2008). The ceramic materials were characterized with thermal-vacuum cycle analysis and Dynamic Mechanical Analysis (DMA) to evaluate their suitability in space environment. Lastly, a preliminary testing was conducted to measure the thrust produced by the ceramic microthruster via electrolytic ignition of HAN propellant.

\section{Materials and methods}

\subsection{Fabrication of ceramic microthruster}

Preparations of ATZ and YSZ-Gr suspensions for gel casting have been described in our previous studies (Markandan et al. 2015, 2017). The microchannel pattern in the thruster was drawn and cut onto adhesive film of $100 \mu \mathrm{m}$ thickness using a cutting plotter (CE6000-60, Graphtec). The film thickness is equivalent to the depth of microstructures. The unwanted parts on the film will be removed, leaving behind a negative pattern which serves as a master mold to prepare a PDMS soft mold, as shown in Fig. 1. The Vinyl film master mold and the replicated PDMS soft mold with microthruster design are shown in Fig. 2. ATZ suspension was then casted into the mold and 


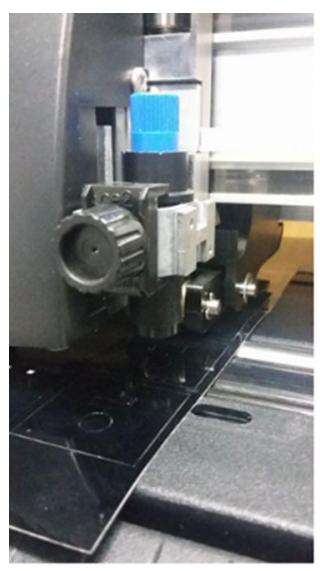

Cut film

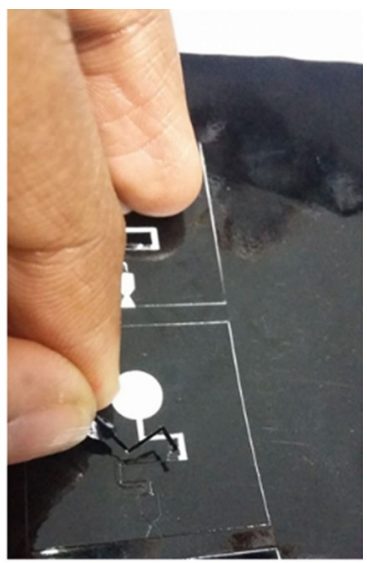

Removal of unnecessary film around pattern
Fig. 1 Preparation of vinyl master mold using cutting plotter

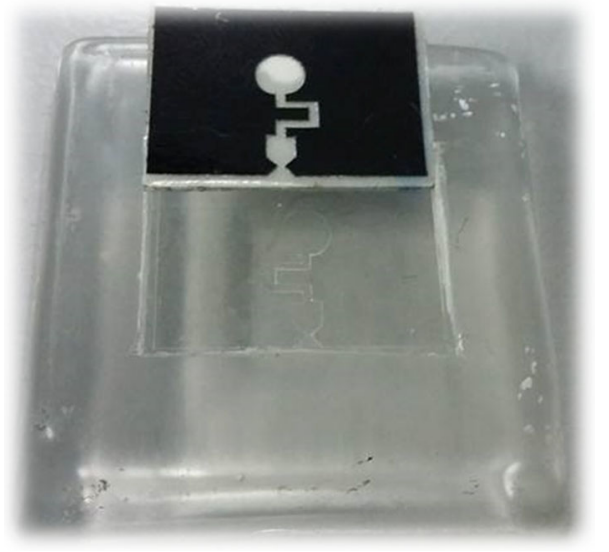

Fig. 2 Vinyl film master mold with geometry of microthruster design and replicated PDMS soft mold

heated to $50{ }^{\circ} \mathrm{C}$ to accelerate gelation process. YSZ-Gr suspension was poured into the remaining region of the mold and reheated to $50{ }^{\circ} \mathrm{C}$.

$85 \%$ aqueous glycerol solution was applied as adhesive to laminate the ceramic green layers, followed by sintering via double step sintering by first ramping the temperature to $1200{ }^{\circ} \mathrm{C}$, reduced to $900{ }^{\circ} \mathrm{C}$ and held for another $30 \mathrm{~h}$ in a furnace (CWF1200, Carbolite). Then, the YSZ-Gr conductive paths were connected to the external electrical circuit and connecting tubes were inserted into the electrode holes and propellant reservoir, respectively. Figure 3 shows the sealed ceramic microthruster with an opening view of the micronozzle exit.
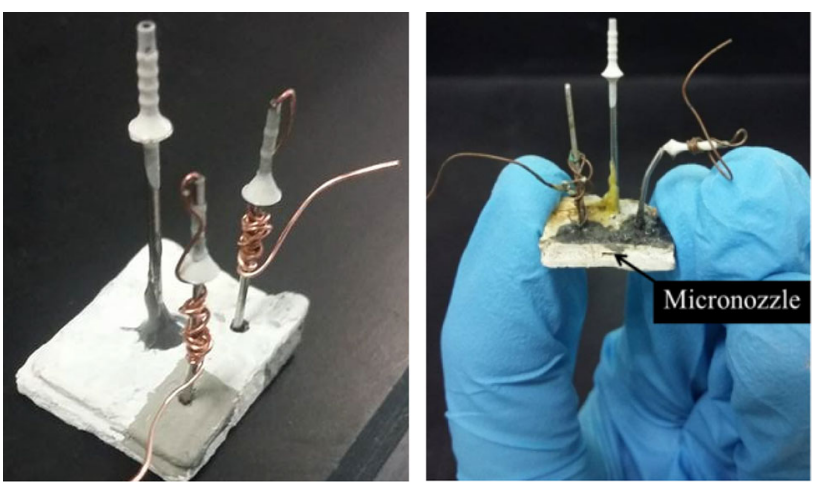

Fig. 3 Sealed microthruster with clear opening view of micronozzle exit

\subsection{Material characterization}

\subsubsection{Thermal-vacuum cycling analysis}

Pieces of ATZ and YSZ-Gr samples with dimension of $1.7 \mathrm{~cm}$ (length) $\times 1.5 \mathrm{~cm}$ (width) $\times 0.5 \mathrm{~cm}$ (thickness) were inserted into a custom-made vacuum chamber. The chamber was connected to a scroll type dry vacuum pump (DIS501, Ulvac Kiko) and a cryogenic pump (CRYO-U 12 HSP). In normal operating condition, the pressure inside the chamber is $8 \times 10^{-5} \mathrm{~Pa}$ with temperature control at $\pm 5{ }^{\circ} \mathrm{C}$. The temperature range was set from 70 to $-20{ }^{\circ} \mathrm{C}$ for a duration of 20 cycles in which each cycle lasted for $120 \mathrm{~min}$.

\subsubsection{Dynamic mechanical test}

Ceramics have excellent refractory properties and high strength that make them ideal candidate for space components. One of the factors impeding the implementation of this material is their poor damping behaviour. The ceramic propulsion device may be subjected to a barrage of shock waves, which impart impulse forces onto the surface generating them. As such, damping of such impulsive forces is vital to prevent accumulative damage. Dynamic mechanical analysis (DMA) is a useful tool to study the damping behaviour of ceramics, in which $\tan \delta$ is used as a measurement of energy dissipated to energy stored.

\subsection{Thrust measurement from the HAN microthruster}

$73 \mathrm{wt} \%$ of hydroxylammonium nitrate (HAN) ionic liquid was used as the propellant in this experiment. It is a green and environmental-friendly liquid propellant which is less toxic, easier handling and storage, as well as clean decomposition byproducts such as oxygen, water and nitrogen. The propellant was synthesised in house, in which 
the process and details of characterization can be found in our previous study (Chai et al. 2016). A syringe pump was used to deliver the HAN propellant into reservoir at controlled flowrates of $20,40,60$ and $80 \mu \mathrm{l} / \mathrm{s}$, respectively. The propellant undergoes electrolytic decomposition using a potential difference of $80 \mathrm{~V}$ and current of $0.1 \mathrm{~A}$. A load cell (Futek, USA) with sampling rate of $100 \mathrm{~ms}$ and accuracy up to $0.01 \mathrm{mg}$ was placed $2 \mathrm{~mm}$ from the micronozzle to measure the thrust generated in $120 \mathrm{~s}$.

\section{Results and discussion}

\subsection{Suitability of material in space environment}

\subsubsection{Thermal-vacuum cycling}

Thermal-vacuum cycling induces repeated stress at the ceramic interface, thereby weakening the bond of ceramic particles. The reduction in the device dimension and weight is less than $1 \%$, showing that most of the volatile chemicals and binders eliminated in multiple stages of heating and sintering. The material hardness has negligible changes after thermal-vacuum cycling while fracture toughness reduces by $9-11 \%$, as shown in Table 1 . The results suggest that both materials can function properly in thermal-vacuum environment.

\subsubsection{Dynamic mechanical analysis}

The addition of graphene platelets in YSZ not only providing electrical conducting paths for electrolytic decomposition, but also enhancing the damping behaviour by energy dissipating mechanism such as GNP pull-outs (Colombo et al. 2010). $\tan \delta$ for different compositions of YSZ-Gr composite are presented in Fig. 4. It can be seen that the presence of graphene platelets improves the damping behaviour of all but slow down towards $100 \mathrm{~Hz}$. This is mainly due to the insufficient recovery time for effective damping behaviour. The effect of $0.5 \mathrm{wt} \%$ graphene platelets in comparison to monolithic YSZ is trivial at all frequencies since there is insufficient layers of graphene to improve the damping behavior. The YSZ reinforced with $1 \mathrm{wt} \%$ graphene platelets shows significant improvement in damping behaviour at low frequency, with improvement of $280 \%$ over monolithic YSZ at a frequency

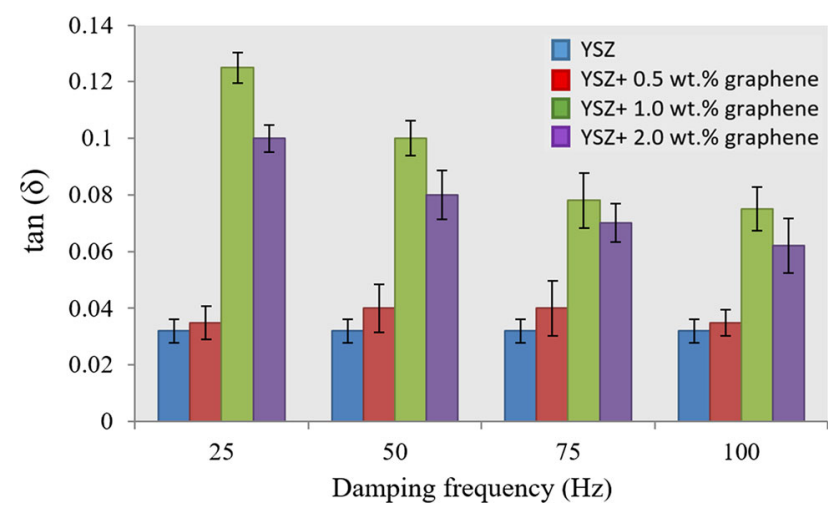

Fig. 4 Damping behaviour (tan $\delta$ ) of YSZ-graphene platelets (GP) composites at different frequencies

of $25 \mathrm{~Hz}$. In our previous study, scanning electron microscopy images revealed that YSZ reinforced with $1 \mathrm{wt} \%$ graphene platelets are widely dispersed and distributed uniformly throughout the sample to form a networked structure with electrical conductivity of $0.9 \mathrm{~S} / \mathrm{cm}$ (Markandan et al. 2016). This composition was chosen in the fabrication of electrode of good electrical conductivity and excellent damping behaviour.

\subsection{Design and fabrication of ceramic microthruster}

The layout of the zirconia composite microthruster design is shown in Fig. 5 with dimension of $1.7 \mathrm{~cm}$ (length) $\times$ $1.3 \mathrm{~cm}$ (width) $\times 0.5 \mathrm{~cm}$ (thickness) and weigh only $6.18 \mathrm{~g}$. The HAN solution was pumped into the reservoir and flows through a microchannel with $90^{\circ}$ bends, before entering the reaction chamber. Electrolytic ignition of HAN solution in the reaction chamber produces gaseous by-products that are accelerated through the micronozzle to produce thrust.

A Hydrazine milli-Newton thrust $(\mathrm{HmNT})$ weighed 40 grams, developed by JPL for high $\Delta V$ manoeuvres in a cubesat $(<3 \mathrm{~kg}$ ) produces $130 \mathrm{mN}$ (Mueller et al. 2008) was used for scale up for microthrusters in nanosatellites. A factor of 3 of scale up shows that approximately $390 \mathrm{mN}$ is required for similar applications in a nanosatellite $(<10 \mathrm{~kg})$.

The micronozzle design in the microthruster is based on a design previously developed in our research graoup. Detailed descriptions on the design processes of
Table 1 Effect of thermal cycling on mechanical properties of the composite

\begin{tabular}{llllllll}
\hline Material & \multicolumn{2}{l}{ Hardness $(\mathrm{GPa})$} & & & \multicolumn{4}{l}{ Fracture toughness $\left(\mathrm{MPam}^{0.5}\right)$} \\
\cline { 2 - 3 } & Before & After & $\%$ & & Before & After & $\%$ \\
\hline YSZ-Gr & $16.60 \pm 0.47$ & $15.62 \pm 0.26$ & -5.9 & & $4.42 \pm 0.23$ & $4.01 \pm 0.31$ & -9.23 \\
ATZ & $15.35 \pm 0.036$ & $14.98 \pm 0.33$ & -2.41 & & $4.96 \pm 0.08$ & $4.43 \pm 0.18$ & -10.67 \\
\hline
\end{tabular}


Fig. 5 a Design of ATZ microthruster. b Green layers of the device after demoulding. Layer 1 is the top layer drilled with three holes and layer 2 is the bottom layer with the microchannel configuration. The microthruster was formed by folding both layer 1 and 2 towards each other. Hence the side walls of the reaction chamber acts as the electrodes
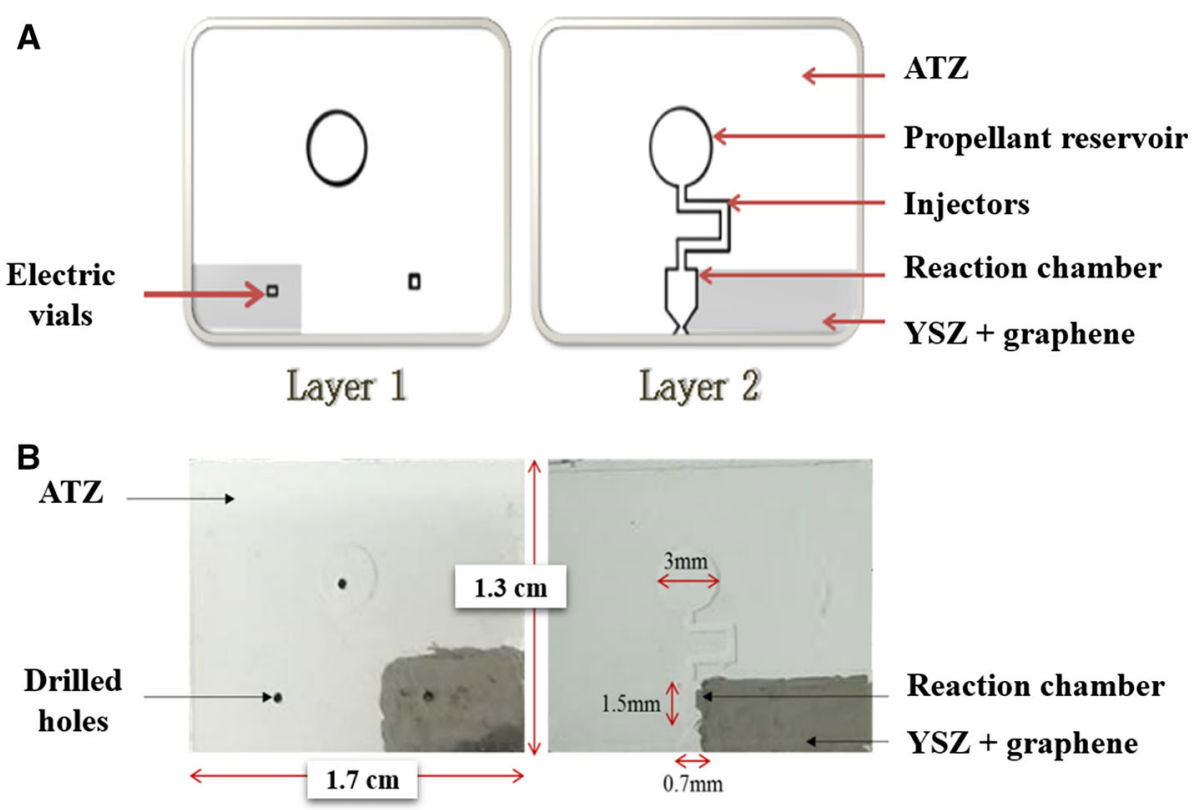

micronozzle, i.e. design consideration, geometrical sizing and performance simulation using CFD are well-documented (Cheah and Chin 2011). Design parameters of the existing micronozzle were revised accordingly and summarized in Table 2, to generate a target thrust of $360 \mathrm{mN}$. The converging part of the nozzle has a half angle of $30^{\circ}$. Expansion ratio of the thruster is 4.4 while the throat depth was kept at $300 \mu \mathrm{m}$. Previous numerical studies (Cheah and Chin 2011) have concluded that higher diverging angle, i.e. $30^{\circ}$, has reduced the viscous loss, which subsequently improve the efficiency of micronozzle operating at low Reynolds number. However, the Reynolds number at nozzle throat has increased to 24,704 as the micronozzle was scaled up for higher thrust, hence the diverging angle of the micronozzle was reduced to the typical value of $15^{\circ}$.

Table 2 Design parameters of the micronozzle

\begin{tabular}{ll}
\hline Design parameter & Value \\
\hline Nozzle depth & $300 \mu \mathrm{m}$ \\
Nozzle throat width & $160 \mu \mathrm{m}$ \\
Nozzle exit width & $704 \mu \mathrm{m}$ \\
Expansion ratio & 4.4 \\
Mass flow rate $(80 \mu \mathrm{l} / \mathrm{s})$ & $1.472 \times 10^{-4} \mathrm{~kg} / \mathrm{s}$ \\
Throat Reynolds number & 24704 \\
Design thrust & $361 \mathrm{mN}$ \\
\hline
\end{tabular}

\subsubsection{Surface contour of ceramic microthruster}

Dispersion of graphene in YSZ composite is critical since the YSZ-graphene paths were used as electrodes. Before sealing the device, both ATZ and YSZ-graphene were examined under SEM to ensure even distribution of graphene and avoid stacking. Figure 6 shows the SEM images of ATZ and a graphene flake among YSZ, respectively. Figure 7 shows different sections of the ATZ microthruster. All design features including the propellant reservoir, combustion chamber, injector and micronozzle were successfully molded. A closer inspection reveals the capability of this technique to produce vertical smooth sidewalls. However, geometrical irregularity was occasionally observed, as shown in Fig. 7a. The presence of irregularities in non-critical components of the thruster is within the tolerance limit.

It is noteworthy that geometrical dimensions of the micronozzle have been upscaled by $15 \%$ from the design values to account for the expected shrinkage of 10-15\% during the sintering process (Cheah and Chin 2013). After comparing the dimension of five (5) micronozzles before and after the sintering, the shrinkage is evaluated as $15 \%$ in average. Quantifying measurements on the nozzle throat width and height after different fabrication processes were conducted and summarized in Table 3.

\subsubsection{Performance evaluation of the microthruster}

As shown in Fig. 8, the thrust profiles at low $(20 \mu \mathrm{l} / \mathrm{s}$ and $40 \mu \mathrm{l} / \mathrm{s})$ and high flowrates $(60 \mu \mathrm{l} / \mathrm{s}$ and $80 \mu \mathrm{l} / \mathrm{s})$ can be divided into 4 stages. First stage refers to a $6 \mathrm{~s}$ initial delay before the electrolytic decomposition of HAN solution 
Fig. 6 SEM images of a ATZ and $\mathbf{b}$ YSZ-graphene surfaces
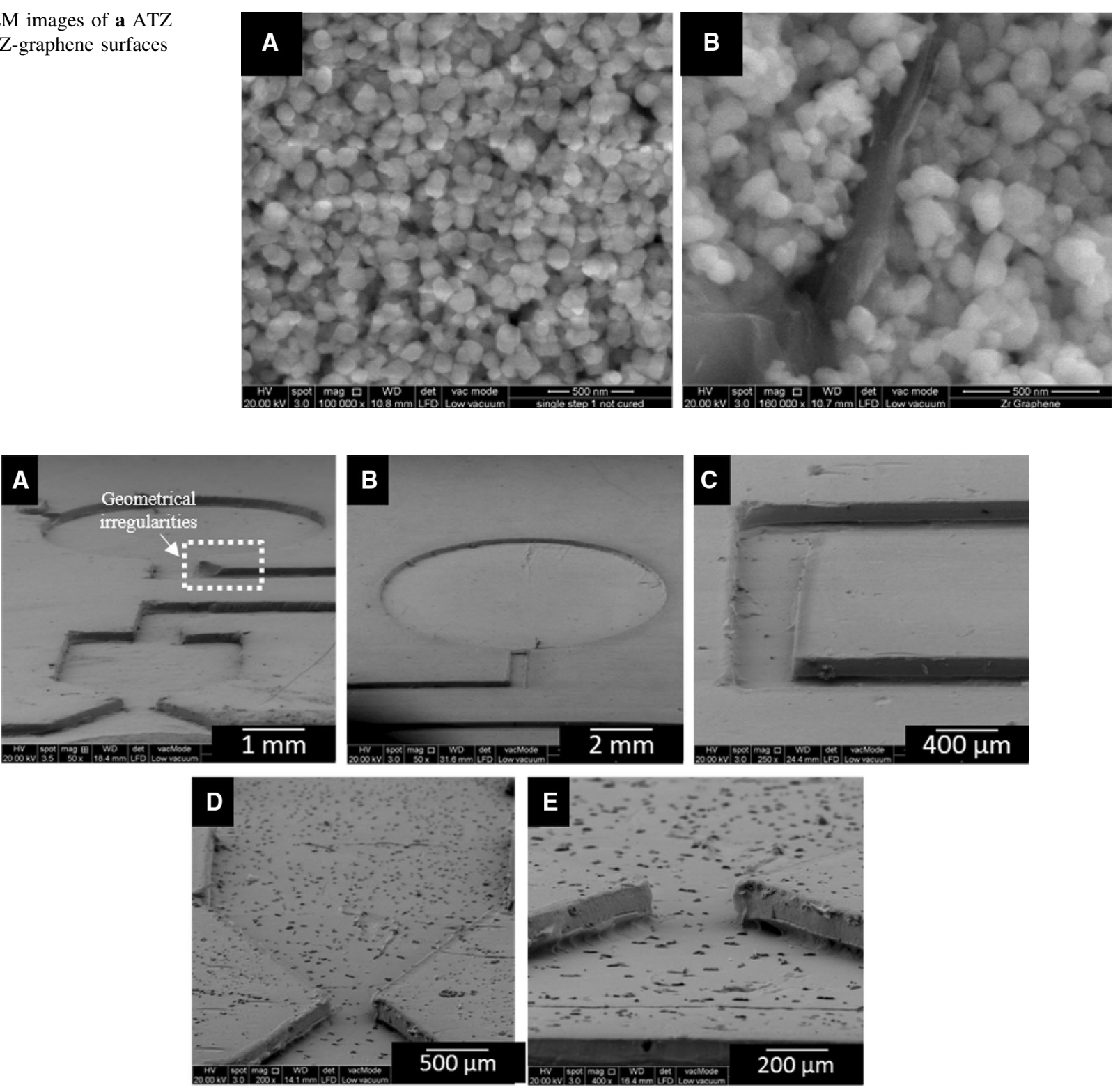

Fig. 7 SEM images of microthruster sections. a The overall microthruster configuration, b propellant reservoir, $\mathbf{c}$ microchannels, $\mathbf{d}$ reaction chamber, e micronozzle

Table 3 Measurements of geometrical dimension of micronozzle throat after different fabrication processes

\begin{tabular}{lcclc}
\hline & Width $(\mu \mathrm{m})$ & $W / W_{\mathrm{o}}(\%)$ & Height $(\mu \mathrm{m})$ & $h / h_{\mathrm{o}}(\%)$ \\
\hline Master mold & $185 \pm 10$ & 100 & $345 \pm 5$ & 100 \\
PDMS mold & $183 \pm 0.5$ & 98.9 & $342 \pm 0.4$ & 99.1 \\
Green ceramic & $176 \pm 0.3$ & 94.9 & $327 \pm 0.3$ & 94.8 \\
After sintering & $156 \pm 0.7$ & 84.3 & $293 \pm 0.3$ & 84.7 \\
\hline
\end{tabular}

starts. The second stage is the thrust generation stage, in which the HAN solution decomposes into hot gases. The thrust level increases steadily until a maximum value is reached. In the third stage, thrust level drops from the maximum value. After a few seconds, the thrust level achieves its steady state.

In this study, the maximum thrust achieved increases proportionally to the flowrate as anticipated, as shown in Fig. 8e. A maximum thrust of $180.53 \mathrm{mN}$ was recorded at flowrate of $60 \mu \mathrm{l} / \mathrm{s}$. However, the steady state thrust remains rather constant at approximately $50 \mathrm{mN}$. This implies that the electrolytic technique is only effective in initiating the decomposition of HAN solution but not able to sustain the reaction when HAN solution was fed into the thruster continuously. The increasing amount of gases 

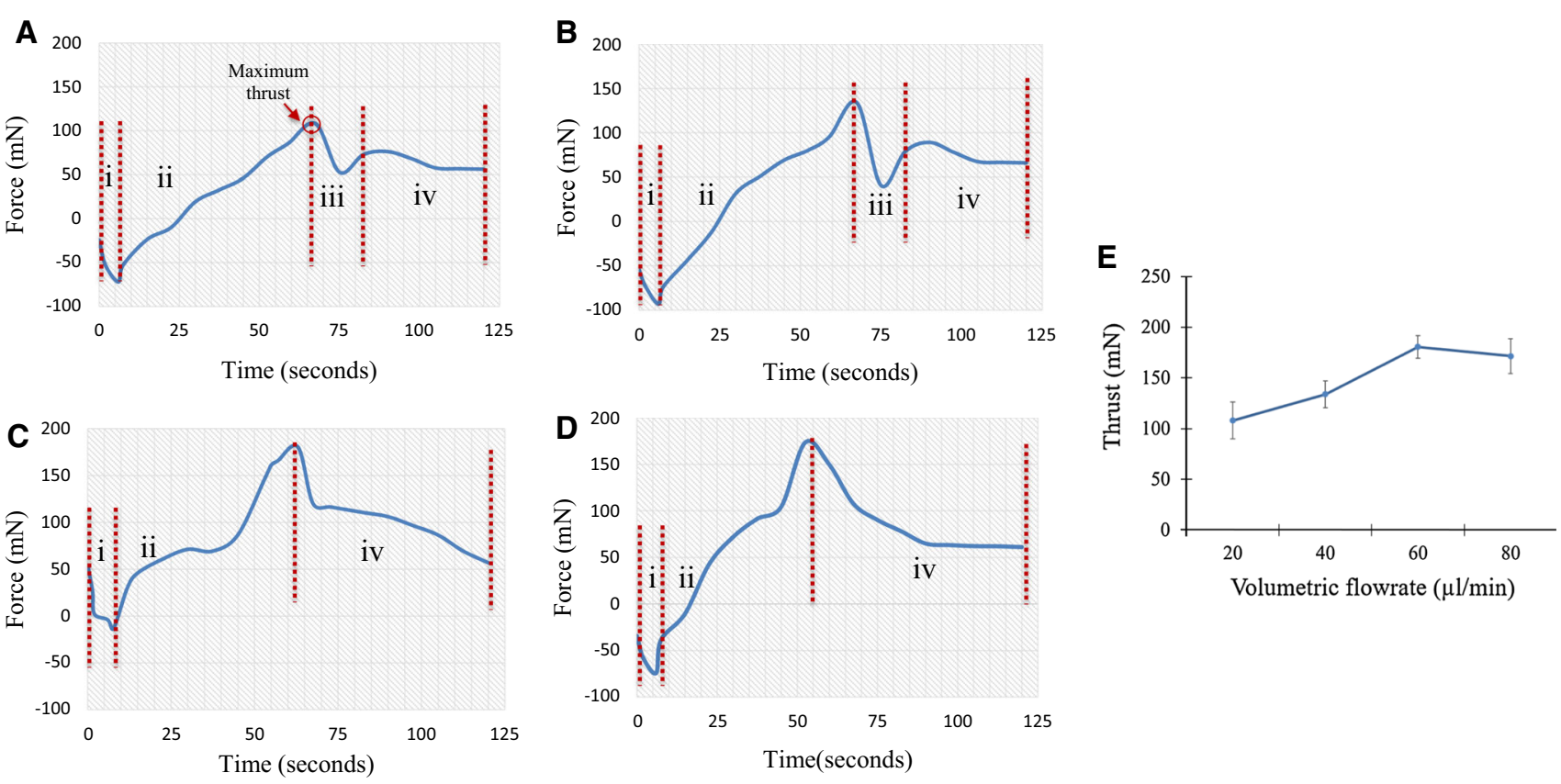

Fig. 8 Thrust profiles of ceramic microthruster obtained at different propellant flowrates a $20 \mu 1 / s$, b $40 \mu 1 / s$, c $60 \mu 1 / s$ and d $80 \mu 1 / s$. e Maximum attainable thrust at different propellant flowrates

produced from electrolytic decomposition has disrupted the continuity in deposition of electrical energy into the HAN solution. This is justified as unreacted HAN solution was observed flowing out from the micronozzle exit during the experiment.

There are two reasons contributing to the thrust fluctuation. First, accumulation and sudden discharge of gas bubbles in the reaction chamber towards the micronozzle due to variation of hydrodynamics condition in the upstream in reaction chamber (Chai et al. 2016). The condition was due to the sinusoidal pumping behaviour of the syringe pump.

The maximum thrust achieved in this study is only $50 \%$ of the design thrust (180.53 mN against $361 \mathrm{mN})$, suggesting the electrolytic decomposition alone is insufficient to achieve complete consumption of the flowing HAN solution. Although using of catalyst is common in macroscaled chemical propulsion system, pre-heating of catalyst bed requires substantial electrical energy, making it unfavourable for microthrusters in nanosatellites. Further improvement could be made to combine the electrolytic and catalytic techniques. Electrolytic ignition technique can be used in the initial stage to partially decompose the HAN solution into warm liquid-gas mixture, followed by flowing into a catalyst bed for complete decomposition to generate thrust.

\subsection{SEM analysis on microthrustrer after electrolytic decomposition}

The SEM analysis was only limited to ATZ but not the YSZ-graphene layer because its area in contact with HAN is limited to a thin layer at the wall. An original ATZ surface is shown in Fig. 9a, with clear and smooth surface. Figure $9 b$, c are two different reaction chamber surfaces after $2 \mathrm{~min}$ and $5 \mathrm{~min}$ of electrolytic decomposition of HAN at $60 \mu 1 / \mathrm{s}$, respectively. Figure $9 \mathrm{~b}$ has some spheres and pits, and appears to be octahedral crystals while the surface fired while Fig. 9c has micron-sized platelets covering the surface. The phenomena on the ATZ samples are similar to that of hot corrosion (Schacht et al. 1998; Park et al. 2005; Manning et al. 2015), in which tetragonal phase of the zirconia was transformed into monolithic phase. The associated microcracks provides paths for minor leakage of gas products which reduces the pressure inside the reaction chamber. As such, this preliminary study indicates that further improvement is necessary to extend the operational lifetime of the ceramic microthruster. The sintering temperature could be increased to $1400-1500{ }^{\circ} \mathrm{C}$. Higher sintering temperature was proved to be effective in reducing the internal porosities and increases the thermo-mechanical properties, e.g. hardness has increased for as high as $20 \%$ for ATZ sintered at $1400{ }^{\circ} \mathrm{C}$ (Kern and Gadow 2012). 

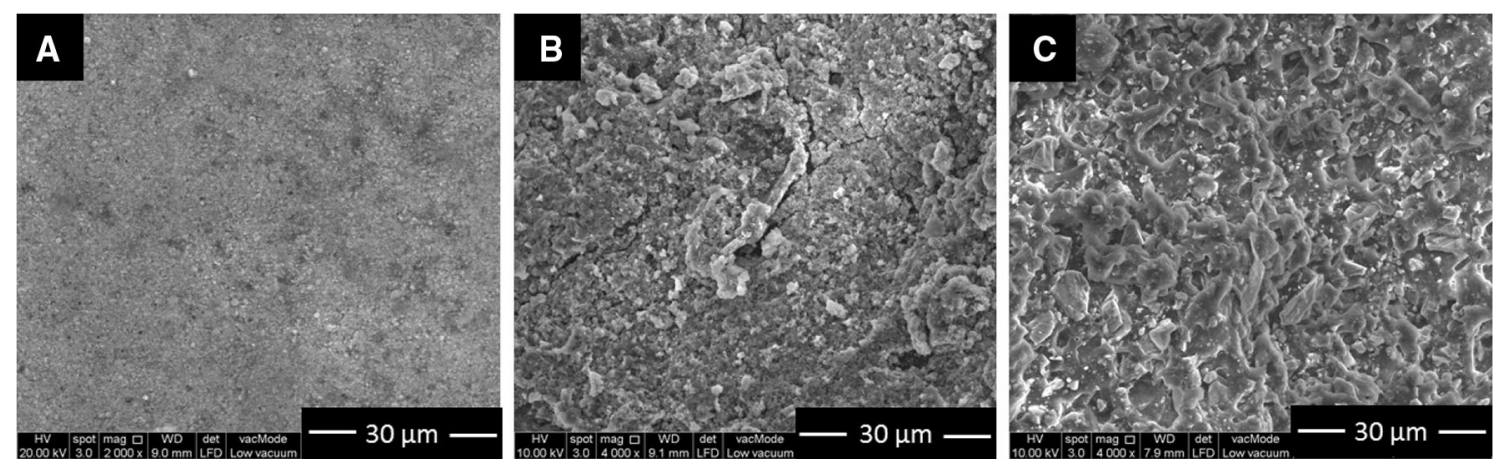

Fig. 9 SEM images of microthruster. a Original ATZ surface. b ATZ surface fired with $60 \mu \mathrm{l} / \mathrm{g}$ propellant for 2 min and c ceramic surface fired with $60 \mu \mathrm{l} / \mathrm{g}$ propellant for $5 \mathrm{~min}$

\section{Conclusions}

In this paper, a microthruster fabricated with ATZ and YSZ-graphene has been demonstrated. YSZ-graphene is not only served as electrodes for electrolytic decomposition of HAN solution but also playing a role in damping. Thermal-vacuum analysis and dynamic mechanical analysis were conducted to evaluate the change in material properties under simulated space environments.

Thrust generated by the ceramic microthruster at different flowrates was measured using a load cell. The maximum thrust achieved is $180.53 \mathrm{mN}$ at flowrate of $60 \mu \mathrm{l} / \mathrm{s}$. The thrust measurement experiment reveals the incomplete decomposition of HAN solution using electrolytic technique alone. Nevertheless, the experimental results suggest that electrolytic technique could be combined with the conventional catalytic technique for a complete combustion of HAN solution which releases more energy and subsequently generates higher thrust.

The ceramic microthruster weights only $6.18 \mathrm{~g}$ and able to generate thrust above $100 \mathrm{mN}$. After the discovered issues in decomposition of HAN solution are addressed, a high thrust-to-weight ratio microthruster could be realized in the future. Such microthruster has potential applications as primary propulsion system for rapid orbital transfer in constellation formation flying of satellites.

Acknowledgements The authors would like to acknowledge University of Nottingham Malaysia Campus for providing facilities.

Open Access This article is distributed under the terms of the Creative Commons Attribution 4.0 International License (http://creative commons.org/licenses/by/4.0/), which permits unrestricted use, distribution, and reproduction in any medium, provided you give appropriate credit to the original author(s) and the source, provide a link to the Creative Commons license, and indicate if changes were made.

\section{References}

Bartolomé JF, Smirnov A, Kurland H-D et al (2016) New $\mathrm{ZrO}_{2} / \mathrm{Al}_{2} \mathrm{O}_{3}$ nanocomposite fabricated from hybrid nanoparticles prepared by $\mathrm{CO}_{2}$ laser co-vaporization. Sci Rep 6:20589. https://doi.org/10. 1038/srep20589

Cai H, Lewis FL, Hu G, Huang J (2017) The adaptive distributed observer approach to the cooperative output regulation of linear multi-agent systems. Automatica 75:299-305

Chai WS, Cheah KH, Koh KS et al (2016) Parametric studies of electrolytic decomposition of hydroxylammonium nitrate (HAN) energetic ionic liquid in microreactor using image processing technique. Chem Eng J 296:19-27. https://doi.org/10.1016/j.cej. 2016.03.094

Cheah KH, Chin JK (2011) Performance improvement on MEMS micropropulsion system through a novel two-depth micronozzle design. Act Astronaut 69(1):59-70. https://doi.org/10.1016/j. actaastro.2011.02.018

Cheah KH, Chin JK (2012) Fabrication of a zirconia MEMS-based microthruster by gel casting on PDMS soft molds. J Micromech Microeng 22(9):95013. https://doi.org/10.1088/0960-1317/22/9/ 095013

Cheah KH, Chin JK (2013) Fabrication of embedded microstructures via lamination of thick gel-casted ceramic layers. Int J Appl Ceram Technol. https://doi.org/10.1111/ijac.12023

Cheah KH, Low KS (2014) Fabrication and performance evaluation of a high temperature co-fired ceramic vaporizing liquid microthruster. J Micromech Microeng 25:015013. https://doi. org/10.1088/0960-1317/25/1/015013

Chong SJ, Bandyopadhyay S, Foust R, Subramanian GP, Hadaegh FY (2016) Review of formation flying and constellation missions using nanosatellites. J Spacecr Rocket 53(3):567-578. https:// doi.org/10.2514/1.A33291

Colombo P, Mera G, Riedel R, Sorarù GD (2010) Polymer-derived ceramics: 40 years of research and innovation in advanced ceramics. J Am Ceram Soc 93:1805-1837

Edwards-Stewart C (2013) NASA's GRAIL spacecraft formation flight, end of mission results, and small-satellite applications. Annu AIAA/USU Conf Small Satell SSC13-X-2

Hu Q, Dong H, Zhang Y, Ma G (2015) Tracking control of spacecraft formation flying with collision avoidance. Aerosp Sci Technol 42:353-364. https://doi.org/10.1016/j.ast.2014.12.031

Kern F, Gadow R (2012) Alumina toughened zirconia from yttria coated powders. J Eur Ceram Soc 32:3911-3918. https://doi.org/ 10.1016/j.jeurceramsoc.2012.03.014

Khaji Z, Klintberg L, Barbade D et al (2016) Alumina-based monopropellant microthruster with integrated heater, catalytic 
bed and temperature sensors. J Phys Conf Ser 757:12025. https:// doi.org/10.1088/1742-6596/757/1/012025

Kristiansen R, Nicklasson PJ (2009) Spacecraft formation flying: a review and new results on state feedback control. Acta Astronaut 65:1537-1552. https://doi.org/10.1016/j.actaastro.2009.04.014

Lekholm V, Persson A, Palmer K et al (2013) High-temperature zirconia microthruster with an integrated flow sensor. J Micromech Microeng 23:55004. https://doi.org/10.1088/0960$1317 / 23 / 5 / 055004$

Liu G-P, Zhang S (2018) A survey on formation control of small satellites. Proc IEEE 106:440-457. https://doi.org/10.1109/ JPROC.2018.2794879

Manning T, Field R, Klingaman K et al (2015) Innovative boron nitride-doped propellants. Def Technol. https://doi.org/10.1016/j. dt.2015.10.001

Markandan K, Tan MTT, Chin J, Lim SS (2015) A novel synthesis route and mechanical properties of $\mathrm{Si}-\mathrm{O}-\mathrm{C}$ cured Yytria stabilised zirconia (YSZ)-graphene composite. Ceram Int 41:3518-3525

Markandan K, Chin JK, Tan MTT (2016) Enhancing electroconductivity of Yytria-stabilised zirconia ceramic using graphene platlets. Key Eng Mater 690:1-5. https://doi.org/10.4028/www. scientific.net/KEM.690.1

Markandan K, Chin J, Tan MTT (2017) Low temperature fabrication and characterization of $\mathrm{Si}-\mathrm{O}-\mathrm{C}$ cured alumina toughened zirconia (ATZ). Mater Today Proc 4:3005-3013. https://doi. org/10.1016/j.matpr.2017.02.183

Mueller J, Ziemer J, Hofer R, Wirz R, O'Donnell T (2008) A survey of micro-thrust propulsion options for microspacecraft and formation flying missions. CubeSat 5th Annual Developers Workshop

Nevarez-Rascon A, Aguilar-Elguezabal A, Orrantia E, BocanegraBernal MH (2009) On the wide range of mechanical properties of ZTA and ATZ based dental ceramic composites by varying the $\mathrm{Al}_{2} \mathrm{O}_{3}$ and $\mathrm{ZrO}_{2}$ content. Int $J$ Refract Met Hard Mater 27:962-970. https://doi.org/10.1016/j.ijrmhm.2009.06.001

Park SY, Kim JH, Kim MC et al (2005) Microscopic observation of degradation behavior in yttria and ceria stabilized zirconia thermal barrier coatings under hot corrosion. Surf Coat Technol 190:357-365. https://doi.org/10.1016/j.surfcoat.2004.04.065

Persson, D'Amico S, Harr J (2010) Flight results from PRISMA formation flying and rendezvous demonstration mission. In: 61st international astronautical congress

Ren W, Sorensen N (2008) Distributed coordination architecture for multi-robot formation control. Robot Autonom Syst 56:324-333. https://doi.org/10.1016/j.robot.2007.08.005

Romero-Diez S, Hantsche L, Pearl J et al (2018) A single-use microthruster concept for small satellite attitude control in formation-flying applications. Aerospace 5:119. https://doi.org/ 10.3390/aerospace5040119

Schacht M, Boukis N, Dinjus E et al (1998) Corrosion of zirconia ceramics in acidic solutions at high pressures and temperatures. J Eur Ceram Soc 18:2373-2376. https://doi.org/10.4028/www. scientific.net/KEM.132-136.1677

Schneider J, Begand S, Kriegel R et al (2008) Low-temperature aging behavior of alumina-toughened zirconia. J Am Ceram Soc 91:3613-3618. https://doi.org/10.1111/j.1551-2916.2008.02700. $\mathrm{x}$

Sun H, Zhao H, Huang K et al (2017) Adaptive robust constraintfollowing control for satellite formation flying with system uncertainty. J Guid Control Dyn 40:1492-1502. https://doi.org/ 10.2514/1.G002396

Underwood CI, Richardson G, Savignol J (2003) In-orbit results from the SNAP-1 nanosatellite and its future potential. Philos Trans R Soc Lond Ser A Math Phys Eng Sci 361(1802):199-203. https:// doi.org/10.1098/rsta.2002.1123

Wu MH, Yetter R (2009) A novel electrolytic ignition monopropellant microthruster based on low temperature co-fired ceramic tape technology. Lab Chip 9:910-916. https://doi.org/10.1039/ B812737A

Zhang Q, Liu HHT (2018) Aerodynamic model-based robust adaptive control for close formation flight. Aerosp Sci Technol 79:5-16. https://doi.org/10.1016/j.ast.2018.05.029

Publisher's Note Springer Nature remains neutral with regard to jurisdictional claims in published maps and institutional affiliations. 